\title{
Storage capacity of labeled graphs
}

\author{
Dana Angluin ${ }^{1 \star}$, James Aspnes ${ }^{1 \star \star}$, Rida A. Bazzi ${ }^{2}$, Jiang Chen ${ }^{3}$, David \\ Eisenstat $^{4}$, and Goran Konjevod ${ }^{2}$ \\ 1 Department of Computer Science, Yale University \\ 2 Department of Computer Science and Engineering, Arizona State University \\ ${ }^{3}$ Google \\ 4 Department of Computer Science, Brown University
}

\begin{abstract}
We consider the question of how much information can be stored by labeling the vertices of a connected undirected graph $G$ using a constant-size set of labels, when isomorphic labelings are not distinguishable. An exact information-theoretic bound is easily obtained by counting the number of isomorphism classes of labelings of $G$, which we call the information-theoretic capacity of the graph. More interesting is the effective capacity of members of some class of graphs, the number of states distinguishable by a Turing machine that uses the labeled graph itself in place of the usual linear tape. We show that the effective capacity equals the information-theoretic capacity up to constant factors for trees, random graphs with polynomial edge probabilities, and bounded-degree graphs.
\end{abstract}

\section{Introduction}

We consider what happens if we replace the linear tape of a standard Turing machine with some fixed finite connected graph. This gives us a way to represent self-organizing systems consisting of many communicating finite-state machines, where at any time, one machine (the location of the Turing machine head) takes a leadership role. Our main question is how much computing power such machines can cooperate to achieve. The answer depends on the inherent storage capacity of the graph, a function of its size (bigger gives more space) and symmetries (more symmetries makes the space harder to exploit).

In more detail, a graph Turing machine consists of an undirected connected graph $G$, each of whose nodes holds a symbol from some finite alphabet, together with a finite-state controller that can move around the graph and update the symbols written on nodes. Because there is no built-in sense of direction on an arbitrary graph, the left and right moves of a standard Turing machine controller are replaced by moves to adjacent graph nodes with a given symbol. If there is no such adjacent graph node, the move operation fails, which allows the controller to test its immediate neighborhood for the absence of particular symbols. If there is more than one such node, which node the controller moves to is chosen arbitrarily. (A more formal definition of the model is given in Section 3.)

\footnotetext{
* Supported in part by NSF grant CCF-0916389.

** Supported in part by NSF grants CNS-0435201 and CCF-0916389.
} 
The intent of this model is to represent what computations are feasible in various classes of simple distributed systems made up of a network of finite-state machines. Inclusion of an explicit head that can move nondeterministically to adjacent nodes (thus breaking at least local symmetries in the graph) makes the model slightly stronger than similar models from the self-stabilization literature (e.g., Dijkstra's original model in [8]) or population protocols [5]; we discuss the connection between our model and these other models in Section 2.

The main limitation on what a graph Turing machine can compute appears to be the intrinsic storage capacity of its graph. For some graphs (paths, for example) the storage capacity is essentially equivalent to a Turing machine tape of the same size. For others (cliques, stars, some trees), the usable storage capacity may be much less, because symmetries within the graph make it difficult to distinguish different nodes with the same labeling. We define a notion of information-theoretic capacity of a graph (Section 4.1) that captures the number of distinguishable classes of labelings of the graph. Essentially this comes down to counting equivalence classes of labelings under automorphisms of the graph; it is related to the notion of the distinguishing number of a graph, which we discuss further in Section 2.3.

The information-theoretic capacity puts an upper bound on the effective capacity of the graph, the amount of storage that it provides to the graph Turing machine head (defined formally in Section 4.2). Extracting usable capacity requires not only that labelings of the graph are distinguishable in principle but that they are distinguishable to the finite-state controller in a way that allows it to simulate a classic Turing machine tape. We show that an arbitrary graph with $n$ nodes provides at least $\Omega(\log n)$ tape cells worth of effective capacity (which matches the information-theoretic upper bound for cliques and stars, up to constant factors). For specific classes of graphs, including trees (Section 7), random graphs with polynomial edge probabilities (Section 9), and boundeddegree graphs (Section 8), we show that the effective capacity similarly matches the information-theoretic capacity.

Notably, these classes of graphs are ones for which testing graph isomorphism is easy. Whether we can extract the full capacity of a general graph is open, and appears to be related to whether graph isomorphism for arbitrary graphs can be solved in LOGSPACE. We discuss this issue in Section 10.

\section{Related work}

\section{$2.1 \quad$ Self-stabilizing models}

A graph Turing machine bears a strong resemblance to a network of finite-state machines, which has been the basis for numerous models of distributed computing, especially in the self-stabilization literature. Perhaps closest to the present work is the original self-stabilizing model of Dijkstra [8], where we have a collection of finite-state nodes organized as a finite connected undirected graph, and at each step some node may undergo a transition to a new state that depends on 
its previous state and the state of its immediate neighbors. The main difference between the graph Turing machine model and this is the existence of a unique head, and even more so, its ability to move to a single neighbor of the current node - these properties break symmetry in ways that are often difficult in classic self-stabilizing systems. A limitation of the graph Turing machine model is the restriction on what the head can sense of adjoining nodes: it cannot distinguish neighbors in the same state, or even detect whether one or many neighbors is in a particular state.

Itkis and Levin [11] give a general method for doing self-stabilizing computations in asynchronous general topology networks. Their model is stronger than ours, in that each node can maintain pointers to its neighbors (in particular, it can distinguish neighbors in the same state). Nonetheless, we have found some of the techniques in their paper useful in obtaining our current results.

\subsection{Population protocols}

There is also a close connection between our model and the population protocol model [5], in which a collection of finite-state agents interact pairwise, each member of the pair updating its state based on the prior states of both agents (see [6] for a recent survey on this and related models). This is especially true for work on population protocols with restricted communication graphs (for example, [3]). Indeed, it is almost possible to simulate a graph Turing machine in a population protocol, simply by moving the state of the head around as part of the state of the node it is placed on, and using interactions with neighbors to sense the local state. The missing piece in the population protocol model is that there is no mechanism for detecting the absence of a particular state in the immediate neighborhood. Although a fairness condition implies that every neighbor will make itself known eventually, the head node has no way to tell if this has happened yet. Urn automata [4], a precursor to the population protocol model in which a finite-state controller manages the population, also have some similarities to graph Turing machines, especially in the combination of a classical Turing-machine controller with an unusual data store.

The community protocol model of Guerraoui and Ruppert $[9,10]$ extends population protocols by allowing agents to store a constant number of pointers to other agents that can only be used in limited ways. Despite these restrictions, Guerraoui and Ruppert show that community protocols with $n$ agents can simulate storage modification machines as defined by Schönhage [16], which consist of a dynamic graph on $n$ nodes updated by a finite-state controller. Such machines can in turn simulate standard Turing machines with $O(n \log n)$ space. The community protocol and storage modification machine models are both stronger than our graph Turing machines because they allow for a dynamic graph, while our machines have to work with the graph they are given. 


\subsection{Distinguishing number}

The distinguishing number $[2] d(G)$ of a graph $G$ is the minimum number of colors needed to color the vertices of $G$ so that $G$ has no color-preserving automorphisms.

If the distinguishing number of a class of graphs is bounded, then we can in principle color the nodes with a distinguishing coloring that uniquely identifies each node based on its position in the graph (though it still may require substantial work to identify a particular node). With a large enough alphabet, we can use a second component of the state to store the contents of a Turing machine tape cell. This would give an information-theoretic capacity for the graph of $\Theta(n)$.

Albertson and Collins [2] show that any graph has distinguishing number $O(\log (|\operatorname{Aut}(G)|))$. This implies that the information-theoretic capacity of the class of graphs with constant-sized automorphism groups is $\Theta(n)$ (the effective capacity may be smaller in some cases). Thus graphs with low informationtheoretic capacity will have large automorphism groups, i.e., lots of symmetry.

Computing distinguishing number exactly appears to be difficult. Some improved characterizations may be found in $[1,15]$.

\section{Graph Turing machines}

Formally, a graph Turing machine is specified by a 4 -tuple $\left(\Sigma, Q, q_{0}, \delta\right)$ where $\Sigma$ is a finite alphabet of tape symbols, $Q$ is a finite set of controller states, $q_{0} \in Q$ is the initial controller state, and $\delta: Q \times \Sigma \times \mathcal{P}(\Sigma) \rightarrow$ $\left(Q \cup\left\{q_{\text {accept }}, q_{\text {reject }}\right\}\right) \times \Sigma \times \Sigma$ is the transition function. We assume that the alphabet $\Sigma$ contains the special blank symbol -. The graph $G$ on which the machine runs and the initial position of the controller $v_{0} \in V(G)$ are supplied separately.

The first argument of the transition function $\delta$ is the current state of the controller, the second argument is the symbol on the current node, and the third gives the set of symbols that appear on one or more of the neighbors of the current node. The output of $\delta$ gives the new state of the controller, the symbol to write to the current node, and the symbol indicating which adjacent node to move to.

The special states $q_{\text {accept }}$ and $q_{\text {reject }}$ are accepting and rejecting halting states, respectively; if the machine enters one of these two halting states, there is no move to a neighboring node. For transitions that do not enter a halting state, we require that the target symbol be present in the immediate neighborhood (i.e., that it is chosen from the set of neighboring symbols). Implicit in this rule is that, in the unusual event that $G$ contains only a single node $v_{0}$ and the set of symbols on neighboring nodes is empty, the machine must halt immediately.

A configuration of a graph Turing machine $\left(\Sigma, Q, q_{0}, \delta\right)$ running on a graph $G$ is a triple $(q, v, s) \in\left(Q \cup\left\{q_{\text {accept }}, q_{\text {reject }}\right\}\right) \times V(G) \times \Sigma^{V(G)}$ where $q$ is the current state of the controller, $v$ is its current position, and $s$ specifies the current 
tape symbol $s_{v}$ on each node $v$ of $G$. A halting configuration is a configuration in which the controller state is either $q_{\text {accept }}$ or $q_{\text {reject }}$; in the former case it is an accepting configuration and in the latter a rejecting configuration.

We consider $\left(G, v_{0}\right)$ to be the input to the graph Turing machine, where $G$ is a graph and $v_{0} \in V(G)$ is the initial node. Given an input $\left(G, v_{0}\right)$, the initial configuration of the machine is $\left(q_{0}, v_{0},\{-\}\right)^{V(G)}$, i.e., the configuration in which the controller starts on node $v_{0}$ in state $q_{0}$ and all nodes contain the blank symbol. As with standard Turing machines, we write $M\left(G, v_{0}\right)$ for the machine $M$ operating on input $\left(G, v_{0}\right)$.

Given a non-halting configuration $(q, v, s)$, let

$$
\left(q^{\prime}, \sigma_{1}, \sigma_{2}\right)=\delta\left(q, s_{v},\left\{s_{u}:(u, v) \in E(G)\right\}\right) .
$$

There is a transition from $(q, v, s)$ to $\left(q^{\prime}, v^{\prime}, s^{\prime}\right)$ if (a) $s_{v}^{\prime}=\sigma_{1}$, (b) $s_{u}^{\prime}=s_{u}$ for all $u \in V(G)-\{v\}$, and (c) $s_{v^{\prime}}^{\prime}=s_{v^{\prime}}=\sigma_{2}$. Note that there may be more than one such transition if there is more than one neighbor $v^{\prime}$ with $s_{v^{\prime}}=\sigma_{2}$, Note further that there are no transitions from a halting configuration.

Given input $\left(G, v_{0}\right)$, a computation path is a sequence of configurations $C_{0}, C_{1}, \ldots$ where $C_{0}$ is the initial configuration and there is a transition from $C_{i}$ to $C_{i+1}$ for each $i$. A graph Turing machine halts on input $\left(G, v_{0}\right)$ if every computation path is finite. A graph Turing machine accepts (rejects) input $\left(G, v_{0}\right)$ if every computation path is finite and ends in an accepting (rejecting) configuration. The running time of a graph Turing machine with input $\left(G, v_{0}\right)$ is the maximum length of any computation path, or $\infty$ if no such maximum exists.

Though most computations of graph Turing machines are inherently nondeterministic, we will call a graph Turing machine deterministic if for any input $\left(G, v_{0}\right)$ it either accepts on all computation paths or rejects on all computation paths. The justification for this unusual usage is that for a deterministic graph Turing machine, the choice of which of several alternative nodes to move to can be made arbitrarily - possibly even according to some deterministic tie-breaking rule (whose inclusion would complicate the model.)

We say that a graph Turing machine $M_{1}$ with input $\left(G_{1}, v_{1}\right)$ simulates a graph Turing machine $M_{2}$ with input $\left(G_{2}, v_{2}\right)$ if there is a mapping from configurations of $M_{1}\left(G_{1}, v_{1}\right)$ to configurations of $M_{2}\left(G_{2}, v_{2}\right)$ such that every transition of $M_{1}\left(G_{1}, v_{1}\right)$ maps to either a transition of $M_{2}\left(G_{2}, v_{2}\right)$ or to a no-op. Often we will have $\left(G_{1}, v_{1}\right)=\left(G_{2}, v_{2}\right)$, with the main differences between $M_{1}$ and $M_{2}$ being that $M_{2}$ is a graph Turing machine extended in some way, such as by adding multiple heads or more built-in storage. The particular case of simulating a standard Turing machine will be used to define effective capacity in Section 4.2.

\section{Storage capacity of graphs}

In this section, we consider the question of how much information can be stored in a given graph. We first look at the information-theoretic capacity bound 
(Section 4.1), then consider how much of this potential capacity can actually be extracted (Section 4.2).

\subsection{Information-theoretic capacity}

The information-theoretic capacity of a graph is just the base 2 logarithm of the number of distinguishable labelings of its nodes, where two labelings are distinguishable if there is no automorphism of the graph that carries one to the other and equivalent otherwise. This quantity is in principle computable using Burnside's Lemma; the number of distinguishable labelings is

$$
L(G)=|X / \operatorname{Aut} G|=\frac{1}{|\operatorname{Aut}(G)|} \sum_{g \in \operatorname{Aut}(G)}\left|X^{g}\right|,
$$

where $X$ is the set of all labelings, $X / \operatorname{Aut}(G)$ is the quotient set of equivalence classes of labelings under automorphisms in $G$, and $X^{g}$ is the set of labelings preserved by a particular automorphism $g$. The information-theoretic capacity $I_{G}$ of $G$ is then the base $2 \operatorname{logarithm} \lg L(G)$ of this quantity.

In practice, computing the number of distinguishable labelings will be easiest for classes of graphs that have no non-trivial automorphisms, or for which the set of automorphisms has a particularly simple structure, such as cliques, stars, or trees. For example, any permutation of the nodes of a clique, or any permutation of the non-central nodes of a star, is an automorphism of the graph. We can map one labeling to another by a color-preserving permutation precisely when each has the same number of nodes with each color (in the case of a star, when this property holds for the leaves and the central nodes have the same color). It follows that an equivalence class can be specified by counting the number of nodes with each color (plus $O(1)$ bits for the central node for a star). In either case we get $\Theta(\log n)$ bits of information.

Graphs with constant distinguishing number (see Section 2.3) or for which a small number of carefully-colored nodes eliminate color-preserving automorphisms will have information-theoretic capacity $\Theta(n)$. An example would be a path; by fixing distinct colors of the endpoints, no color-preserving automorphisms remain.

The information-theoretic capacity of general trees depends heavily on the structure of the tree: whether it looks more like a star, with many automorphisms, or a path, with few. We discuss this issue in detail in Section 7.

In general, we can bound the information-theoretic capacity of any graph with $n$ nodes by $O(n)$; this is just the number of bits needed to represent all possible labelings without considering equivalence.

The usefulness of the information-theoretic capacity is that it puts an upper bound on how much state can be stored in the graph. Call two configurations of a graph Turing machine equivalent if

1. The head is in the same state in both configurations. 
2. There is a label-preserving automorphism of $G$ that carries the position of the head in the first configuration to the position of the head in the second configuration.

It is not hard to see that equivalent states have equivalent successors, since the same automorphism can be used after a transition as long as we are careful to make the heads move to matching locations. It follows that for the purpose of simulating a graph Turing machine, we need only record its state up to equivalence.

Theorem 1. Fix a graph Turing machine, and suppose it is used to simulate a standard Turing machine. When running on graph $G$ with $n$ nodes and information-theoretic capacity $I_{G}$, the simulation has at most $O\left(I_{G}\right)$ space.

Proof. We can describe a state of the graph Turing machine up to equivalence by specifying (a) some member of a class of equivalent graph labelings $\left(I_{G}\right.$ bits); (b) the state of the finite-state controller $(O(1)$ bits); and (c) the position of the finite-state controller ( $\log n$ bits). Summing these quantities gives $O(1)+$ $\log n+I_{G}$ bits, which translates into at most $O\left(\log n+I_{G}\right)$ tape cells for the simulated machine. But now observe that any graph has $I_{G}=\Omega(\log n)$ (provided the alphabet size is at least 2), since we can obtain at least $n+1$ distinct automorphism classes by labeling $k$ nodes with one symbol and $n-k$ with another, where $k$ ranges from 0 to $n$. So $O\left(\log n+I_{G}\right)=O\left(I_{G}\right)$.

\subsection{Effective capacity}

Our intent is that the effective capacity of a graph is the size of the largest standard Turing machine tape that can be simulated using the graph. However, we can in principle make this size arbitrarily large for any fixed graph by increasing the size of the alphabet and the number of states in the finite-state controller. To avoid this problem, we define effective capacity only for classes of graphs.

Definition 1. A class of graphs $\mathcal{G}$ has effective capacity $f(G)$ if there is a function $f$ such that for any standard Turing machine $M$, there is a graph Turing machine $M^{\prime}$ where for any $G$ in $\mathcal{G}$, and any vertex $v_{0}$ of $G, M^{\prime}\left(G, v_{0}\right)$ simulates $M$ running on an initially blank tape with $f(G)$ tape cells.

Note that because we have not specified alphabet sizes, effective capacity is defined only up to constants. Furthermore, any particular construction can only demonstrate a lower bound on effective capacity. For example, we can show that the class of all graphs has effective capacity $\Omega(\log n)$, where $n$ is the number of nodes in the graph. The reason for this is we can use the nodes in the graph as a unary counter, then use a standard construction [14] to simulate a $\log (n)$ space Turing machine. However, this does not exclude the possibility that some subclass of the class of all graphs has higher effective capacity, or that there might be a construction that obtains $\Omega(\log n)$ space on general graphs while doing better on some specific graphs. 
An example of a class of graphs with high effective capacity are paths. The essential idea is that we can use a path directly to simulate a standard Turing machine tape, with each node in the path representing one cell in the tape. A minor complication is that a standard Turing machine can tell its left from its right while a graph Turing machine can only do so if the neighbors of the current cell have different labels. But we can handle this by adding an extra field in each node that holds repeating values $\{0,1,2\}$ (this is the slope mechanism from [11]), with the left neighbor of a node with value $x$ being the one with $(x-1) \bmod 3$ and the right being the one with $(x+1)$ mod 3 . This extra information triples the size of the alphabet, but that is permitted by the definition.

On the other hand, we can't do any better than $\Theta(n)$. It is immediate from Definition 1 and Theorem 1 that no class of graphs has an effective capacity that exceeds the information-theoretic capacity by more than a constant factor.

Definition 1 also does not include a time bound. A natural restriction would be to consider polynomially-bounded effective capacity, where the simulation can use at most a polynomial number of steps for each step of the simulated machine. In our constructions, we are more interested in showing possibility rather than specific time bounds, but we will state time bounds when we can.

\section{Graph traversal}

A fundamental tool for doing computation with a graph Turing machine is the ability to traverse every node in the graph. In this section, we show how this can be done regardless of the structure of the graph.

We adapt depth-first search to our needs. Depth-first search requires a stack, which we can represent by marking the nodes that are on the stack. Unfortunately, because the graph may contain cycles, a simple mark does not suffice to indicate unambiguously a stack node's parent. If each node were labeled with its distance modulo three from the root, then the correct parent node would be evident from the labels. In order to establish these distance labels, we use depth-first traversal repeatedly on the previously labeled portion of the graph to expand the search by one step at a time from the root, as in breadth-first search.

For the depth-first search method, we assume that every node has the fields color (which may be black, white or gray) and depth (which may be $\infty, 0,1$ or 2 ). The depth field is calculated modulo 3 to keep the size of the state finite; this approach is similar to the "centered slope" technique used in [11]. The variable head refers to the node that is the current position of the graph Turing machine. In the initial configuration in which every node contains the blank symbol, we have $v$.depth $=\infty$ and $v$.color $=$ white for every node $v$. In order to initiate the process of assigning the depth labels, we set head.color to white and head.depth to 0 .

Because depth-first search will be used in the process of establishing the correct depth labels, its invariant refers to $C$, the portion of the graph that has been successfully depth labeled so far. A node $v$ is in $C$ if and only if $v$.depth $\neq \infty$. 
The depth-first search algorithm is a framework to allow the performance of some action at every node of $C$. The action can occur in pre-order or post-order with respect to the search.

During the depth-first search, a node $v$ is finished if $v$.color $=$ black or $v$.depth $=\infty$. A per-node action is safe if it respects the invariant concerning the depth field, leaves the head where it found it, and alters the color or depth fields of a node only when that node is finished and remains finished after the alteration.

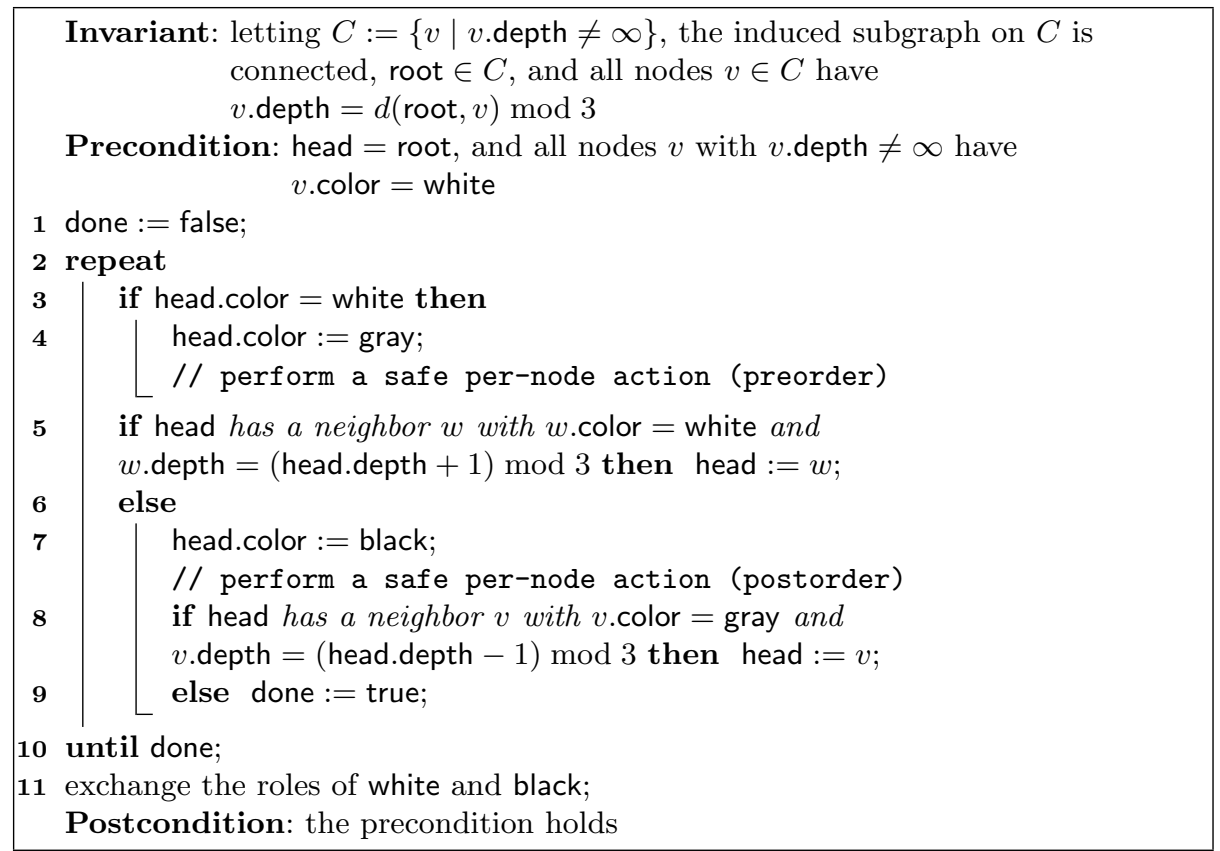

Algorithm 1: Depth-first traversal of a distance-labeled subgraph

In each phase of the breadth-first algorithm to establish the depth labels we use depth-first search to visit every node $v$ in $C$ and perform the safe per-node operation of changing the depth field of every neighbor $w$ of $v$ with $w$.depth $=\infty$ to $(v$.depth +1$) \bmod 3$. If at least one node has its depth field modified, then $C$ has expanded, and the next phase of the breadth-first expansion ensues. Otherwise, the process of assigning depth labels to all nodes accessible from the root is complete.

To establish the correctness of the depth-first search, we observe that at any time, the induced subgraph on the nodes that are white or gray is connected, and the gray nodes are the nodes along a shortest path from the root to the location of the head or a predecessor of it. Because the head never moves to a black node, it is clear that the only way a node fails to have a white or gray 


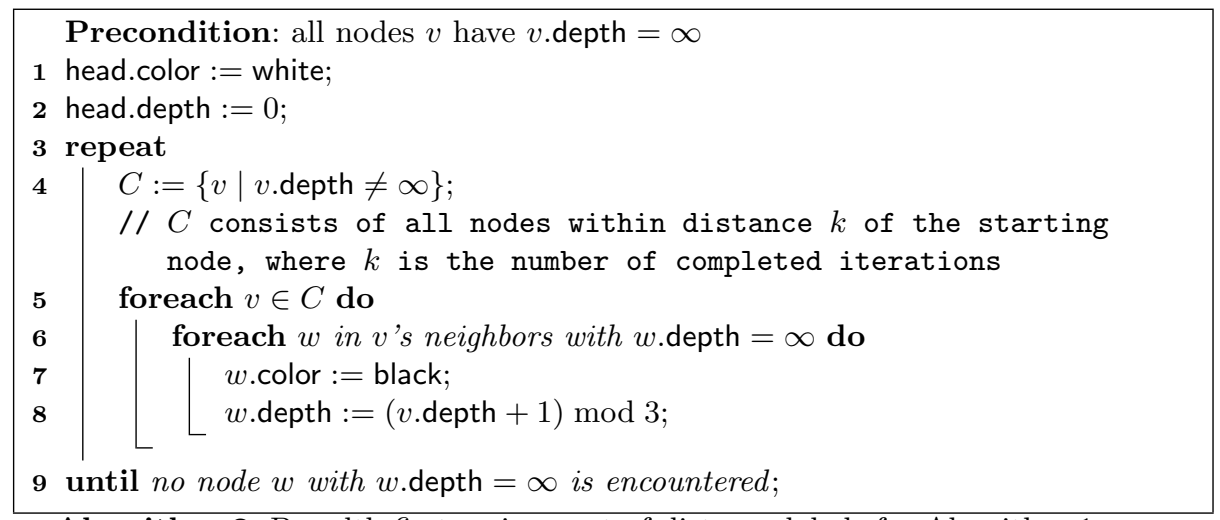

Algorithm 2: Breadth-first assignment of distance labels for Algorithm 1

neighbor is if it is the root and it is the last node to be finished. This is the safety property; the liveness property is that we continue to make progress. If we continue to go down, eventually there must be a node with no white or gray children, which will be made black. This in turn establishes the correctness of the breadth-first depth labeling process: at each phase all the currently labeled nodes are visited, and any unlabeled neighbors of them are properly labeled. All accessible nodes are properly depth labeled if and only if no neighbors of labeled nodes are unlabeled.

If the graph $G$ has $n$ nodes and $m$ edges accessible from the root, the depth labeling process completes in $O\left(n^{2}\right)$ steps. Once all accessible nodes are depth labeled, then the depth-first search process can visit every node in $O(m)$ steps.

\section{Variants of the model}

In the full paper, we show that the graph Turing machine model is robust against minor changes, including:

- Expanding the store on the controller to $O(\log n)$ bits. This space is represented in unary across the nodes of the graph, combining the traversal methods of Section 5 with the classic counter-based Turing machine simulation of Minsky [14].

- Replacing the single head with $k$ heads.

- Removing the controller's ability to see adjacent nodes. Instead, the controller feels its way through the graph "blindly," staying put when it attempts to move to a non-existent neighbor.

These are analogous to classic Turing machine results showing that small changes in the definition do not affect what we can compute. 


\section{Trees}

The question of whether the information-theoretic capacity of a general family of graphs is necessarily achievable effectively seems to be tied up with the problem of graph isomorphism. For trees, the isomorphism problem is simpler and can be solved in polynomial time [13]. Moreover, it is possible to place a total order on classes of isomorphic trees, which can in turn be used to drive a counter simulation that extracts the full storage capacity of the graph, albeit at the expense of an exponential slowdown introduced by the embedded counter machine simulation.

The canonization algorithm of [13] runs in LOGSPACE, so it is tempting to use the LOGSPACE simulation of Section 6 to execute it directly. Unfortunately, the algorithm assumes that the tree is presented on a read-only work tape using unique identifiers for each node. We don't have this in our model. So instead we describe a new mechanism for comparing labeled trees that works despite this restriction, while allowing us to compute the next labeling of a tree in place. Isomorphism and increment can be shown to run in polynomial time.

We consider rooted trees. In the full paper, we show that this does not change the asymptotic storage capacity of the tree.

\subsection{Information-theoretic capacity of a tree}

Let $T$ be a tree and let $T_{1}, T_{2}, \ldots, T_{d}$ be the subtrees rooted at the children of the root of $T$. Let $f_{k}$ map a tree to the number of inequivalent labelings over an alphabet of size $k \geq 1$. Then we have the recurrence

$$
f_{k}(T)=k \prod_{i}\left(\begin{array}{c}
c_{i}+f_{k}\left(U_{i}\right)-1 \\
f_{k}\left(U_{i}\right)-1
\end{array}\right)
$$

where $U_{1}, U_{2}, \ldots, U_{\ell}$ are the non-isomorphic classes of the $T_{j} \mathrm{~s}$, and $c_{i}$ is the multiplicity of $U_{i}$ among the $T_{j}$ s. Note that the base case is provided by the tree with one node, whose root has no children.

\subsection{Comparing trees}

We define an ordering on (isomorphism classes of) labeled rooted trees by induction. Let $T$ be a tree with root label $\ell$ and immediate subtrees $T_{1}, \ldots, T_{c}$; let $T^{\prime}$ be a tree with root label $\ell^{\prime}$ and immediate subtrees $T_{1}^{\prime}, \ldots, T_{d}^{\prime}$. If $\ell<\ell^{\prime}$, then $T<T^{\prime}$. If $\ell>\ell^{\prime}$, then $T>T^{\prime}$. Otherwise, let $C=\left\{T_{1}, \ldots, T_{c}\right\}$ and $D=\left\{T_{1}^{\prime}, \ldots, T_{d}^{\prime}\right\}$ be multisets. If $C=D$, then $T=T^{\prime}$, where subtree equality is defined inductively. If $\max (C-D)>\max (D-C)$, then $T>T^{\prime}$. Otherwise, $\max (D-C)>\max (C-D)$, and $T<T^{\prime}$. It is straightforward to verify that this relation $\leq$ on labeled rooted trees is reflexive, transitive, and antisymmetric up to isomorphism.

In the full paper, we present an algorithm that computes this total ordering. Here, we give only a brief overview of the algorithm. The algorithm assumes a 
graph Turing machine with two heads, one on tree $T$ and one on tree $T^{\prime}$. Each node has a "removed" bit, which is initially unset. The main invariant is that removing all subtrees whose removed bits are set does not affect the order.

The algorithm begins by comparing the root labels. Assuming that they are equal, for each of the immediate subtrees $T_{i}$ of $T$, we attempt to find a immediate subtree of $T^{\prime}$ isomorphic to $T_{i}$. If there is such a subtree $T_{j}^{\prime}$, we set the removed bit for both $T_{i}$ and $T_{j}^{\prime}$; these subtrees offset one another and will not be considered again. If no match can be made, then we set the removed bit for each $T_{j}^{\prime}$ that was determined to be less than $T_{i}$, as these subtrees cannot be the maximum in the symmetric difference of the two multisets of subtrees. At the end, if all immediate subtrees have been removed, then $T=T^{\prime}$. Otherwise, if all immediate subtrees of $T^{\prime}$ have been removed, then $T>T^{\prime}$, else $T<T^{\prime}$.

\subsection{Implementing counters with labeled trees}

In this section we simulate counter machines with operations of clear, increment, and compare counters for equality, which can implement a standard Turing machine, albeit with exponential slowdown [14].

Given a tree $T$ whose nodes can be labeled $0, \ldots, k-1$, we represent counter values $0, \ldots, f_{k}(T)-1$ by the labelings they index in the order defined above. Zero corresponds to the all-zeros labeling, so clearing a register can be accomplished with one traversal. Two counters whose underlying unlabeled trees are isomorphic can be compared using the isomorphism algorithm. The increment operation requires a new algorithm.

We assume that each node of the tree has space to save its old label, and the increment routine will save the previous counter value. To increment a subtree $T$ with overflow, first save the root label and then increment its immediate subtrees (saving their respective values). If every subtree overflowed (i.e., is zero), then increment the root label $\bmod k$ and overflow if it becomes zero. Otherwise, use the isomorphism checker to find and mark the minimum nonzero immediate subtree $M$. Restore every tree other than $M$ to its original value and then zero those that are less than $M$. The call stack for this recursive algorithm is represented using a state label at each node of the tree.

We prove the correctness of the increment algorithm by induction. Suppose we are incrementing a labeled tree $T$ with immediate subtrees $T_{1} \geq \ldots \geq T_{c}$. Let $T^{\prime}$ be the resulting tree. In case $T_{1}, \ldots, T_{c}$ are already at their respective maximums, it is straightforward to verify that $T^{\prime}$ is the successor of $T$. Otherwise, let $U$ be any relabeling of $T$ such that $U>T$. We show that $T<T^{\prime} \leq U$ and thus that $T^{\prime}$ is the successor of $T$.

Let $T_{1}^{\prime} \geq \cdots \geq T_{c}^{\prime}$ be the immediate subtrees of $T^{\prime}$ and let $U_{1} \geq \cdots \geq U_{c}$ be the immediate subtrees of $U$. Note that, on account of the labelings, $T_{i}^{\prime}$ (respectively $U_{i}$ ) may not correspond to $T_{i}$. Given that some $T_{i}$ is not maximum, then the root labels of $T$ and $T^{\prime}$ are identical, and the algorithm is able to find a minimum incremented tree $T_{j}^{\prime}$, where we choose $j$ to be as large as possible in case of ties. We have $T_{i}=T_{i}^{\prime}$ if $i<j$, and $T_{j}<T_{j}^{\prime}$ (count the number of trees greater than or equal to $T_{j}^{\prime}$ ). For all $i>j$, the tree $T_{i}^{\prime}$ is zero. If the root label 
of $U$ is not equal to the root label of $T$ then $T^{\prime}<U$. Otherwise, let $\ell$ be the least index for which $T_{\ell}<U_{\ell}$. For all $i<\ell$, we have $U_{i}=T_{i}$. If $\ell<j$, then $U>T^{\prime}$. Otherwise, $U_{\ell}$ has the same shape (disregarding labels) as some tree $T_{i}<T_{j}^{\prime}$. Since $T_{j}^{\prime}$ was the minimum increment, it follows that $T_{j}^{\prime} \leq U_{\ell}$ and thus that $T^{\prime} \leq U$.

\section{Capacity of a bounded-degree graph}

For a bounded-degree graph, we can use the mechanism in [3] (which itself derives much of its structure from the previous construction in [11]) with only a few small modifications.

In a graph with degree bound $\Delta$, it is possible to assign each node a label in $\left\{1, \ldots, \Delta^{2}+1\right\}$ so that each node's label is unique within a ball of radius 2 . This is a distance two labeling, and it gives each node the ability to identify its neighbors uniquely. Angluin et al.. [3] construct a distance two labeling nondeterministically, by having each node adopt a new label if it detects a secondorder neighbor with the same label. In our model, we can construct the labeling deterministically, by iteratively assigning each node a label that does not conflict with a second-order neighbor (it is easy to see that each time we do this, we cannot create any new conflicts, so we converge after $O(n)$ iterations to a correct labeling).

Using such a labeling, it is straightforward to adapt the traversal routine to build a spanning tree, which in turn can simulate a Turing machine tape to provide $\Theta(n)$ bits of effective capacity.

\section{$9 \quad$ Random graphs}

Suppose we consider random graphs $G$ drawn from $G(n, p)$, i.e., a graph on $n$ nodes where each edge $u v$ appears with probability $p .^{5}$ Suppose further that $p$ scales as $\Theta\left(n^{-c}\right)$ for some fixed $0<c<1$. Then it is possible to achieve an effective capacity of $\Theta(n)$ with high probability ${ }^{6}$ from graphs in this class. Note that graphs in this class are connected with high probability.

The basic idea is that if we can compute a total order on nodes, we can use each node to hold one Turing machine cell, with left and right movements corresponding to moving down or up in the ordering. We compute this ordering by assigning a signature to each node, based on random values stored in its neighborhood. For simplicity, we assume that the Turing machine simulator can generate random values. However, we suspect that a more sophisticated application of the same basic approach could work using only the randomness inherent in the graph.

\footnotetext{
${ }^{5}$ See $[7,12]$ for an overview of random graphs.

${ }^{6}$ We use with high probability to mean that the probability that the event does not occur is $O\left(n^{-c}\right)$ for any fixed $c$.
} 
Details are given in the full paper. The key step in the proof is to show that, if every node in $G(n, p)$ with $p=n^{-c}$ is labeled with a random bit, then the probability that two nodes $u$ and $v$ have the same number of neighbors with 1 bits is $O\left(n^{(c-1) / 2}\right)$, which can be reduced below $O\left(n^{-3}\right)$ by repeating the construction $k=O(1)$ times. The resulting $k=O(1)$ neighborhood counts then give a unique signature for almost all nodes with high probability, which can be computed and compared easily by a LOGSPACE controller.

The full result is:

Theorem 2. A member of the family of random graphs $G(n, p)$ where $p=$ $\Theta\left(n^{-c}\right)$ for any fixed $0<c<1$ has effective capacity $\Theta(n)$ with high probability.

\section{Conclusion}

We have defined a new class of graph-based Turing machines, motivated by potential applications in self-organizing systems of finite-state automata. We have shown that this class is robust under natural changes to the model, and that its power is primarily characterized by the effective capacity of the underlying graph, which is the amount of usable storage obtained by writing symbols from a finite alphabet on its nodes. This is at least $\Omega(\log n)$ bits of space for an arbitrary $n$-node graph, and rises to $\Theta(n)$ bits for bounded-degree graphs and almost all random graphs with polynomial edge probabilities. For trees, the effective capacity ranges from $\Theta(\log n)$ for trees with many symmetries (stars) to $\Theta(n)$ for trees with few (binary trees, paths). In intermediate cases we have shown that we can always get within a constant factor of the full information-theoretic capacity corresponding to the number of non-isomorphic states, although the time complexity of our algorithm could be significantly improved.

The main open problem remaining is whether it is possible to extract the full information-theoretic capacity from an arbitrary graph. This seems closely

tied to the problem of computing graph isomorphism, which is not known to be hard, even for LOGSPACE. The reason is that distinguishing two different labelings of a graph appears to depend on being able to distinguish between nonisomorphic subgraphs (since this gives a weak form of orientation to the graph). However, the problem is not exactly the same, because we have the ability to supplemental isomorphism testing by using some of our labels as signposts and we do not need a perfect isomorphism tester as long as we can group subgraphs into small equivalence classes. So it may be that extracting the full capacity of an arbitrary graph is possible without solving graph isomorphism in general.

\section{Acknowledgments}

The authors would like to thank Yinghua Wu for many useful discussions during the early stages of this work. 


\section{References}

1. Michael O. Albertson. Distinguishing Cartesian powers of graphs. Electronic Journal of Combinatorics, 12:N17, 2005.

2. Michael O. Albertson and Karen L. Collins. Symmetry breaking in graphs. Electronic Journal of Combinatorics, 3(1):R18, 1996.

3. Dana Angluin, James Aspnes, Melody Chan, Michael J. Fischer, Hong Jiang, and René Peralta. Stably computable properties of network graphs. In Proc. Distributed Computing in Sensor Systems: 1st IEEE International Conference, pages 63-74, 2005.

4. Dana Angluin, James Aspnes, Zoë Diamadi, Michael J. Fischer, and René Peralta. Urn automata. Technical Report YALEU/DCS/TR-1280, Yale University Department of Computer Science, November 2003.

5. Dana Angluin, James Aspnes, Zoë Diamadi, Michael J. Fischer, and René Peralta. Computation in networks of passively mobile finite-state sensors. Distributed Computing, 18(4):235-253, March 2006.

6. James Aspnes and Eric Ruppert. An introduction to population protocols. In Benoît Garbinato, Hugo Miranda, and Luís Rodrigues, editors, Middleware for Network Eccentric and Mobile Applications, pages 97-120. Springer-Verlag, 2009.

7. B. Bollobás. Random Graphs. Cambridge University Press, second edition, 2001.

8. Edsger W. Dijkstra. Self-stabilizing systems in spite of distributed control. Communications of the ACM, 17(11):643-644, 1974.

9. Rachid Guerraoui and Eric Ruppert. Even small birds are unique: Population protocols with identifiers. Technical Report CSE-2007-04, Department of Computer Science and Engineering, York University, 2007.

10. Rachid Guerraoui and Eric Ruppert. Names trump malice: Tiny mobile agents can tolerate byzantine failures. In Susanne Albers, Alberto Marchetti-Spaccamela, Yossi Matias, Sotiris E. Nikoletseas, and Wolfgang Thomas, editors, ICALP (2), volume 5556 of Lecture Notes in Computer Science, pages 484-495. Springer, 2009.

11. G. Itkis and L. A. Levin. Fast and lean self-stabilizing asynchronous protocols. In Proceedings 35th Annual Symposium on Foundations of Computer Science, pages 226-239, 1994.

12. Svante Janson, Tomasz Łuczak, and Andrezej Ruciński. Random Graphs. John Wiley \& Sons, 2000.

13. Steven Lindell. A logspace algorithm for tree canonization (extended abstract). In STOC, pages 400-404. ACM, 1992.

14. M. L. Minsky. Computation: Finite and infinite machines. Prentice-Hall series in automatic computation. Prentice-Hall, Inc., Englewood Cliffs, N.J., 1967.

15. Alexander Russell and Ravi Sundaram. A note on the asymptotics and computational complexity of graph distinguishability. Electronic Journal of Combinatorics, 5(1):R23, 1998.

16. Arnold Schönhage. Storage modification machines. SIAM J. Comput., 9(3):490508, 1980. 